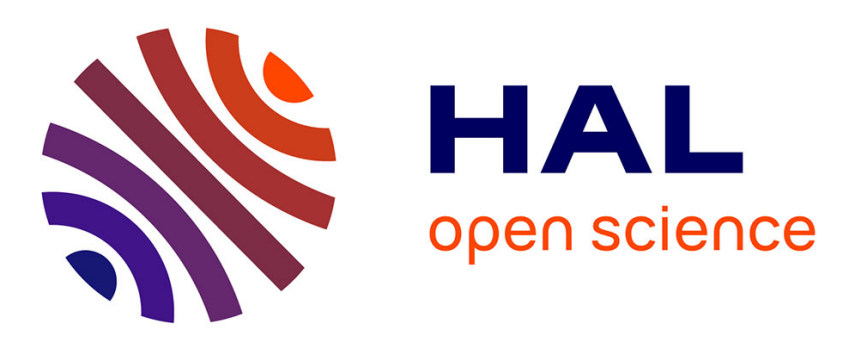

\title{
Robotic Micro-assembly of microparts using a piezogripper.
}

David Hériban, Michaël Gauthier

\section{To cite this version:}

David Hériban, Michaël Gauthier. Robotic Micro-assembly of microparts using a piezogripper.. IEEE/RSJ International Conference on Intelligent Robots and Systems, IROS'08., Sep 2008, Nice, France. pp.4042-4047. hal-00326308

\section{HAL Id: hal-00326308 https://hal.science/hal-00326308}

Submitted on 2 Oct 2008

HAL is a multi-disciplinary open access archive for the deposit and dissemination of scientific research documents, whether they are published or not. The documents may come from teaching and research institutions in France or abroad, or from public or private research centers.
L'archive ouverte pluridisciplinaire HAL, est destinée au dépôt et à la diffusion de documents scientifiques de niveau recherche, publiés ou non, émanant des établissements d'enseignement et de recherche français ou étrangers, des laboratoires publics ou privés. 


\title{
Robotic Micro-assembly of Microparts Using a Piezogripper
}

\author{
David Hériban, Michaël Gauthier IEEE Member \\ FEMTO-ST Institute, UMR CNRS 6174 - UFC / ENSMM / UTBM \\ Automatic Control and Micro-Mechatronic Systems Department \\ 24, rue Alain Savary, 25000 Besançon, FRANCE \\ david.heribandfemto-st.fr, michael.gauthierafemto-st.fr
}

\begin{abstract}
This paper deals with robotic micro-assembly of silicon micro-objects whose sizes are tens of micrometers. This production means is one of a more promising approach to realize 3D and/or hybrid microsystems. Current works in robotic micro-assembly are focused on the assembly of microobjects on a large substrate. We are focusing in the study of micro-parts assembly to build microscopic subsystems usable in larger products. This approach requires specific functionalities like a 'micro-vise' required to block the first object during assembly. Original strategies are proposed and applied on an experimental robotic structure composed of micropositionning stages, videomicroscopes, piezogripper, and silicon endeffectors. Some experimental teleoperated micro-assemblies has validated the proposed methods and the reliability of the principles. Future works will be focused on micro-assembly automation.
\end{abstract}

\section{INTRODUCTION}

In a general way, design of Micro Electromechanical Systems (MEMS) are restricted to monolithic structures which can be realized directly by microfabrication. In fact, the microfabrication capabilities highly reduces diversity and functionalities of microsystems. Innovative ways are required to build new generation of out of plane and/or hybrid microsystems [1], [2].

In the macroworld, building complex and hybrid systems requires assembly to simplify fabrication processes of each product's components. As robotic capabilities were not able to perform reliable assembly of micro-parts, this production means was not consider for MEMS in the first place. The micro-assembly has required study of micromanipulation strategies and robotic design adapted to the microworld and especially to the surface and adhesion forces [3], [4]. In these last five years, micro-assembly's performances has grown and this approach is now consider as a future means of MEMS fabrication.

Two micro-assembly approaches are considered: the selfassembly and the robotic assembly. The self-assembly consists in using bulk objects and to control assembly by a physical field. Each object tend to move to minimum of physical field energy. By using a right physical field, selfassembly is able to position a lot of objects in a parallel way. However, most of self-assembly methods are proposed for objects up to $1 \mu \mathrm{m}$ or down to $100 \mu \mathrm{m}$ [5], [6], [7]. One

This work is supported by the french research project PRONOMIA ANR $n^{\circ}$ 05-BLAN-0325-01 of the major drawback of these methods is that it requires bulk stock of objects. For small object (up to $1 \mu \mathrm{m}$ ), bulk stock can be considered, especially in liquid where colloidal suspension can be obtained. In colloidal suspension, objects are not in contact and can be positioned by self-assembly without adhesion perturbations [5]. For large object (up to $100 \mu \mathrm{m}$ ), adhesion and surface forces are most of time negligible compared to weight or inertia, and bulk stock can be considered too [6], [7]. Between these two physical limits, bulk stock of objects induces contact of micro-objects which can not be easily separated before (or during) self-assembly. In this dimensional domain, the robotic approach seems to be the best way to perform reliable assembly [1], [2]. Some hybrid approaches which combine both methods are also studied [8].

One of the major stakes in robotic assembly is the ability to grasp, position and release a micro-object (usually defined as 'micromanipulation'). Non contact methods can be considered (laser trapping, DEP, etc.), they are able to position objects without adhesion perturbations [9], [10]. However, they cannot induce large blocking forces and thus cannot be considered in a lot of assembly process (insertion, lock, etc.). In other hand, the contact microhandling can be divided into two groups: (i) the passive grippers and the active grippers. In passive gripping, objects must have a specific imprint to be grasped by the passive gripper, release is obtained by using a specific imprint on the substrate (clip, lock, etc.) [1], [2]. Both substrate and objects must have specific imprints dedicated to grasping and release. The active grippers have one or two fingers, the grasp is thus obtained respectively by adhesion or by clamping. The release is performed using specific repulsive forces (inertial release, DEP release, etc.) [11], [12]. As the trajectory of the object after release cannot be controlled, these strategies are able to grasp a microobject but cannot position it with a sufficient precision. Moreover, current efficiency of these release strategies stays low. Consequently, the only way able to position microobjects with a sufficient precision and a large blocking force is currently the passive grippers. However, these methods are not able to manipulate a large type of objects because the design of the object are highly constrained by the imprint required for grasping and release.

We are proposing new reliable methods to manipulate and assemble micro-objects without specific imprint. To 
guarantee a large blocking force which is required in a lot assembly process, we chose to use a two fingers gripper. Contrary to current works, our proposed release strategies are able to position the micro-object with a good repeatability and reliability. Moreover, current works in robotic assembly are focused in the study of micro-parts' assembly on a millimetric substrate [1], [2]. These works are a first step, where assembled systems are millimetric objects. Our proposed approach is focused on possible ways to obtain a microscopic assembly product. Then microproduct could be used as a subsystem in a larger one. This approach is based on the ability to assemble at least two microparts and requires specific micromanipulation strategies dedicated to this problem. This article deals with the feasibility of this approach based on the description of two micro-objects assembly performed in teleoperation.

The section II is dedicated to the original assembly strategies. The whole experimental robotic system is presented in section III. The two last sections deal with the positioning and the assembly of micro-parts.

\section{Assembly Strategy}

We propose to assemble micro-parts in two steps. The first one consists in positioning the first object and blocking it during assembly. The objective of the second step is to grasp the second object and perform assembly. Both steps require robotic capabilities (Degree of Freedom, repeatability...) presented in the section III and specific strategies adapted to the microworld presented in the following.

\section{A. First Micro-object Micromanipulation Principle}

We are proposing a new reliable and reversible method to position micro-object on a substrate. The principle is an hybrid strategy between adhesion manipulation and gripping and is based on a hierarchy of forces. In one hand, to guarantee object's release, the adhesion force between object and substrate must be higher than the adhesion force between object and gripper along the normal vector $\vec{n}$ of the substrate (see in figure 1(a)):

$$
F_{\text {object-substrate }}^{\text {adhesion }} \gg F_{\text {object-gripper }}^{\text {adhesion }}
$$

To reduce the impact of external perturbations, $F_{\text {object-gripper must be lower as possible and }}^{\text {adhesion }}$ $F_{\text {object-substrate }}^{\text {adhesion }}$ must be higher as possible. The major drawback of this release method is the difficulties to grasp the object on the substrate [13]. A reliable grasping cannot be obtained by using only the adhesion force of the gripper. This method is a good way to release the object but not for grasping.

In other hand, to grasp the object, a gripping force higher than the adhesion force between substrate and object along the direction $\vec{n}$ is required (see in figure $1(\mathrm{~b})$ ):

$$
F_{\text {object-gripper }}^{\text {gripping }} \gg F_{\text {object-substrate }}^{\text {adhesion }}
$$

One of the best technological solution is to use gripper with two fingers where the gripping force could be easily higher than adhesion between the object and the substrate.
Our hydrid method uses advantages of both adhesion manipulation and gripping. It induces a reliable release and grasping of micro-object. To guarantee, the conditions (1, 2 ), the gripper must have a high ratio between its gripping force and the adhesion force 'object-gripper'. Technological solutions is proposed in section IV.

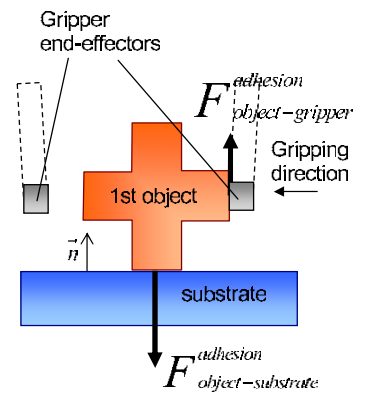

(a) Releasing the first object on the substrate

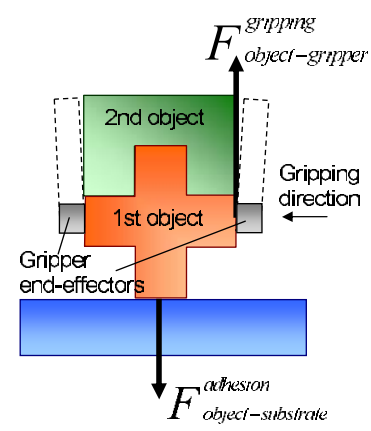

(b) Grasping the first object from the substrate
Fig. 1. Principle of the Release and Grasping of the First Object

\section{B. Micro-assembly of Both Objects}

Two points have to be considered to perform robotic micro-assembly: to guarantee sufficient repeatability, accuracy and compliance, during the assembly and to not disturb the assembly during second object release.

The repeatability and accuracy of the robotic structure are discussed in the section III.

The compliance is one of the major stakes in assembly and especially in the microworld (see figure 2(a)). Without compliance, both objects have to be position with a very high accuracy typically up to hundred of nanometers for a microscopic object. By using compliance, both objects could be position with a lower accuracy, typically around one micrometer. Compliance can be obtained by mechanical elastic structures on the object [2], on the gripper [1], or on the substrate [14].

The three solutions have advantages and drawbacks. In a general way, the component (object, gripper, or substrate), where the compliance is realized, requires usually complex microfabrication capabilities. When the elastic structure is on the object, the object is by definition specific and microassembly principle cannot be extended to other objects [2]. As we are focused on a generic micro-assembly principle, we do not consider this approach. Elastic structures could be consequently placed on the gripper or on the substrate. The gripper requires degrees of freedom, microfabricated end-effectors and is a more complex component than the substrate. To split up the design constraints into both components, we chose to realize the compliance on the substrate. 
Like the first object, the release of the second one requires a specific strategy. Two cases can be considered :

- Both objects have to be locked during assembly. In this case, both objects can be considered as the same object, and the release strategy of the first object (see in figure 1(a)) can be used.

- Both objects do not have to be locked during assembly. It could be the case, in the construction of a larger product, where for example a third object is used to lock the whole assembly. In this case, the previous strategy cannot be used. We are proposing to work on the gripper trajectory to be able to release the second object without adhesion perturbation. An example of trajectory is proposed in figure $2(\mathrm{~b})$.

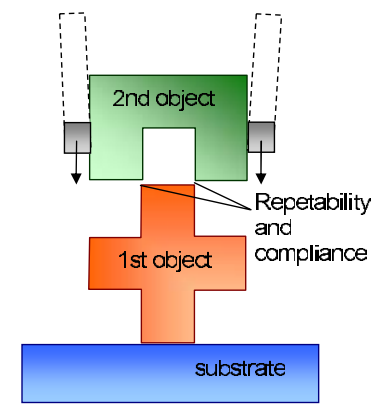

(a) Positioning of the second object before assembly

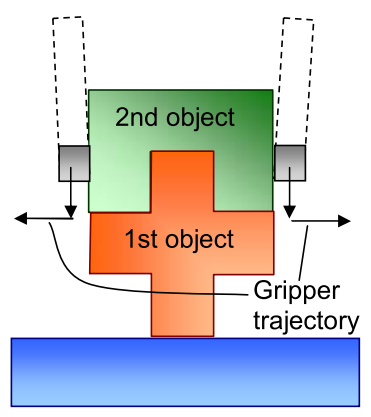

(b) Release of the second object
Fig. 2. Principle of the Positioning, Assembly and Release of the Second Object

The implementation and experimentation of these strategies are presented in the following sections.

\section{ROBOTIC MICRO-ASSEMBLY DEVICE}

The robotic micro-assembly device is composed of a robotic structure, optical microscope, a piezogripper. These elements and the micro-objects used are presented in this section.

\section{A. Robotic structure}

Performing serial micro-assembly tasks requires adapted robotic structures, able to position micro-objects with sufficient accuracy and repeatability, typically up to $1 \mu \mathrm{m}$ for microparts whose typical size is about $10 \mu \mathrm{m}$. These performances are mainly reachable by closed-loop robotic microstages. Nevertheless, in case of complex robotic structure with a gripping device, robotic joint sensors are not sufficient to determine micro-object positioning. Then, using a videomicroscope with a dedicated vision computer is an important way to perform closed-loop control on the entire robotic structure, including the microgripper. Moreover, it allows teleoperated control of the robot by a human operator.

Then, the presented robotic structure (figure 3) is composed by three linear micropositioning stages (Physik Instrumente - M111.1DG). The robot is divided into two

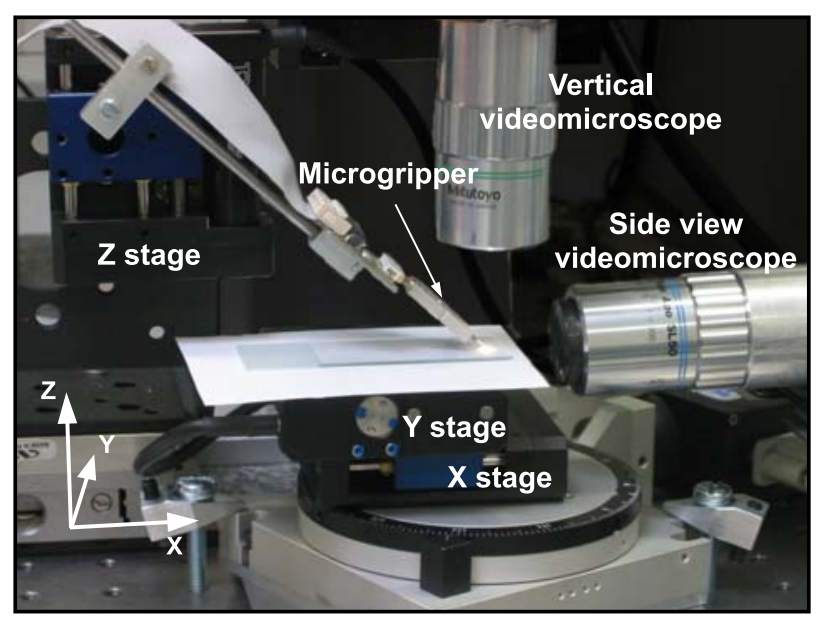

Fig. 3. Robotic device

independent mobile structures. The first part is the robot's arm, a vertical stage carrying the microgripper. The second part, composed by two stages on $\mathrm{X}$ and $\mathrm{Y}$ axis, is used to move micro-objects under a vertical videomicroscope. A lateral view is also added to allow manual teleoperations. The robotic structure is build to get videomicroscopes motionless: the vertical view is focalized on the substrate and the lateral view is focalized on the gripper's end-effectors (see in figure $3)$.

\section{B. Piezoelectric Microgripper}

The MMOC piezomicrogripper[15] used in this robotic structure was developed in our laboratory. It has 2 independent degrees-of-freedom for each fingers, which can perform open-close motion of $320 \mu \mathrm{m}$ and up-down motion of 200 $\mu \mathrm{m}$. The resolution of the actuator is close to $1,6 \mu \mathrm{m} / \mathrm{V}$ then submicrometric accurate motions are controllable. Several kind of finger tips can be glued on this piezoelectric actuator. Up-down motion of gripper's actuator is in fact uses to align them before manipulation. The finger tips[16] used for micro-assembly have been designed to handle microscopic objects. They are build in single crystal silicon SOI wafer by a well-known microfabrication process: DRIE. These endeffectors have a long and thin beam $(12 \mu \mathrm{m})$ designed to handle objects from $5 \mu \mathrm{m}$ to few hundred micrometers.

\section{Design of Micro-objects}

Testing micro-assembly needs micro-objects that could be mechanically fastened to the others. Thus, micro-objects have been designed with mechanical fastener structures already studied in[17]. To supply a challenging benchmark, objects' shape are squares of $40 \mu \mathrm{m}$ sides with a thickness of 5 $\mu \mathrm{m}$. SOI wafers of $5 \mu \mathrm{m}$ device layer thickness and DRIE process have been used to build these microparts. Many shapes, fastener designs and sizes were tested (figure 4). Two kind of parts are presented in this article: the first one is 40 $\mu m$ square puzzle parts, with four notches of $5 \mu \mathrm{m}$. The second one is a mechanical plug device between two $40 \mu \mathrm{m}$ 
squares. The male part have a key which is able to lock the female part after assembly as proposed by Dechev[17].

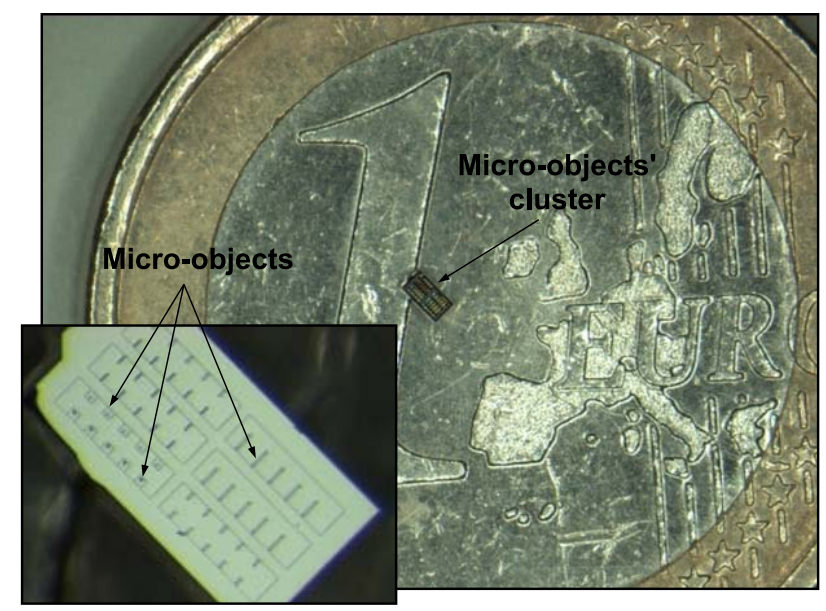

Fig. 4. Micro-objects designed for assembly.

\section{FIRST OBJECT POSITIONING}

\section{A. Adapting adhesive effects}

As presented in figure 1, two ways have been chosen to guarantee first object's manipulation: increase adhesion forces between the substrate and the object and decrease adhesion force between the object and the gripper.

We chose to use as substrate a transparent gel film wellknown in microelectronics: Gel-Pak. This material is in fact transparent and softly adhesive, it consequently allows accurate pick and place tasks. Moreover, the low mechanical stiffness of this polymer induces natural compliance of the substrate required for micro-assembly. In a second time, efforts have been made on end-effectors shaping. First, surface in contact with the micro-object has been reduced by using end-effectors with a small thickness. In second time, the fabrication process called DRIE have been used to give the gripping surface a specific texture. Etching anisotropy of this process is made by a short succession of isotropic etching/protection cycles. These cycles create a phenomenon called scalloping illustrated in figure 5. In this way, contact shape between object and end-effectors is a succession of microscopic contact points. As proved by [18], the roughness induced by DRIE is able to highly reduce pull-off force. Force measurements will be performed in a near future to validate the surface force reduction, and the adhesion of the Gel-Pak.

\section{B. Pick and place}

A micro-object is placed on the substrate. First, gripper is moved above and fingers are opened enough to grip the object. Then the object is hold by the end-effectors and gripper is use to separate the object from the substrate. Currently, our gripper has no force sensors and the gripping force is so not controlled yet. The substrate is moved to a new position (target position). Finally, release is performed

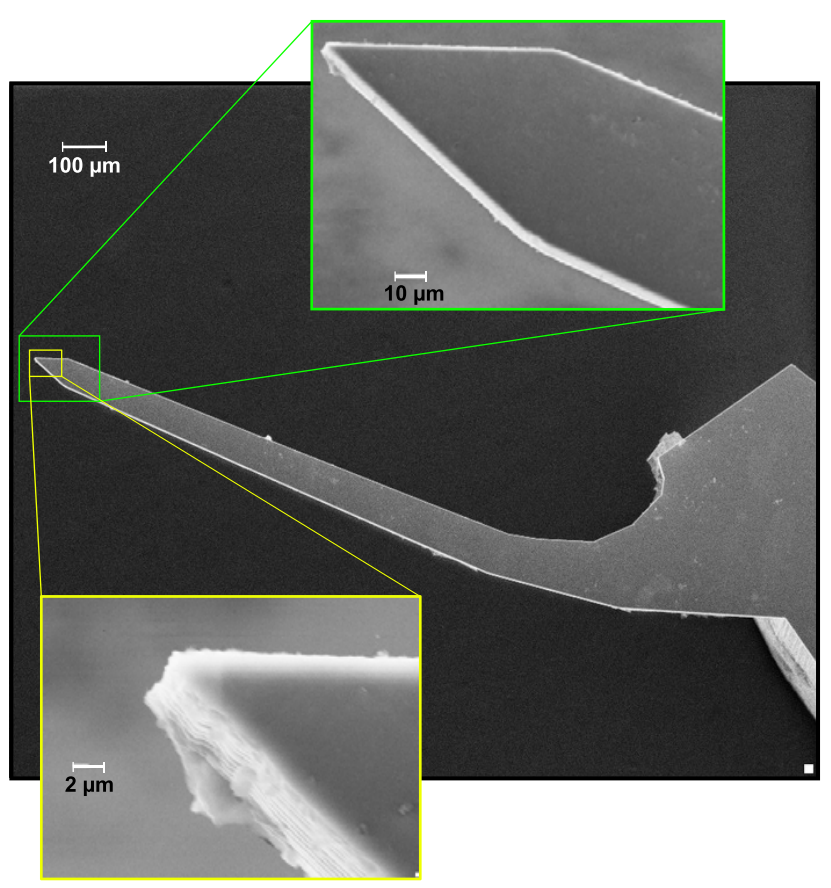

Fig. 5. End-effectors' shape in SEM view. Scalloping is visible in lower picture.

by moving down the gripper to create a contact between object and adhesive substrate then opening gripper induces the release of the object. All the micromanipulation sequence is shown in figure 6 .
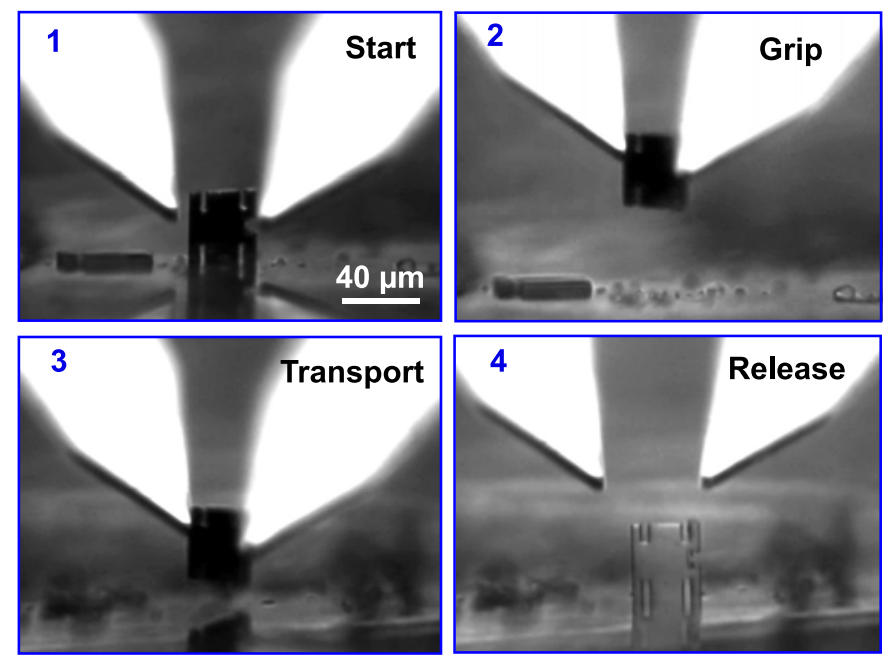

Fig. 6. Pick and place of $40 \mu m$ micro-objects.

Without adhesive substrate (eg. on silicon or glass), it is very difficult to release object because during the gripper opening, the micro-object still stick on one of both endeffectors.

\section{EXPERIMENTAL MiCRO-ASSEMBly}

Robotic agility of the presented micro-assembly station has been tested in teleoperate mode to assemble benchmark 
micro-objects. Two kind of mechanical assembly have been tried to make a three-dimensional microproduct. The first one is made by an insertion of two identical puzzle parts. The second one is a reversible assembly of two different parts.

\section{A. Insertion}

Each puzzle piece has four notches, close to $5 \mu \mathrm{m}$ width and $10 \mu \mathrm{m}$ long. As part's thickness is $5 \mu \mathrm{m}$, assembly of two pieces requires to insert perpendicularly (figure 7).
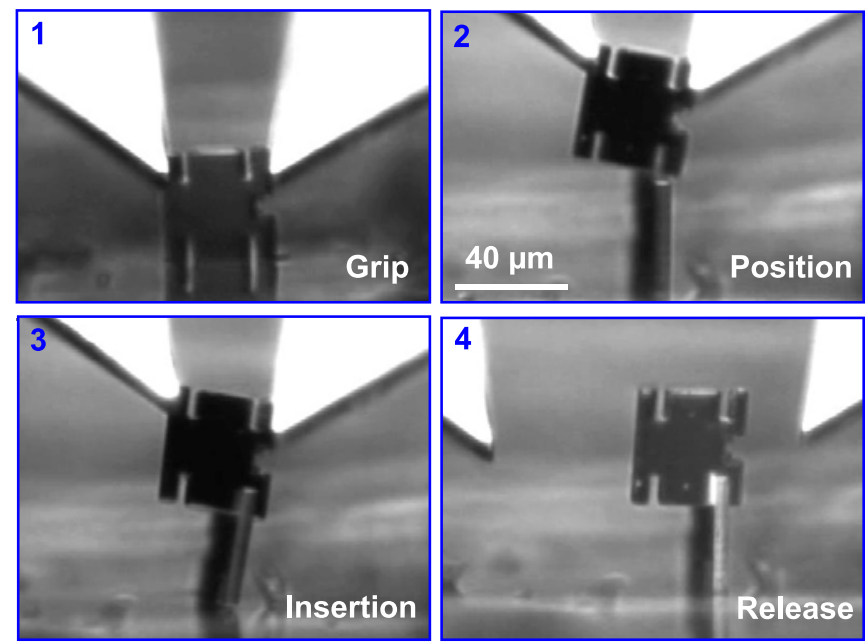

Fig. 7. Insertion assembly.

The first part is gripped and place vertically on the substrate. The second part is taken vertically too perpendiculary to the first one (step 1). Then two puzzle pieces are ready to be assembled. Then the second part is gripped, and is accurately positioned above the first part (step 2). Assembly clearance is very small and evaluated to $200 \mathrm{~nm}$ by SEM measurement and accuracy can be made up by substrate compliance. Indeed, compliance of adhesive substrate allows small rotative motion of the first part thus insertion is easily performed without any fine orientation of the gripper (step 3 ). When insertion is complete, microgripper is opened to release assembled part (step 4). This last operation can failed when adhesive effects between gripper and puzzle piece are stronger than between both puzzle pieces. In fact, the part stay sticked on the end-effector and opening the gripper disassemble the micro-product. Consequently, the trajectory proposed on section II is used to induce a reliable release.

\section{B. Reversible Assembly}

The second assembly benchmark requires more steps and more accuracy. Both mechanical parts are different but have the same square shape of $40 \mu \mathrm{m}$ side. The first part have a small key joint with a $\mathrm{T}$ shape on one side. The second part have a $\mathrm{T}$ shaped imprint in center of the square (figure 8). To perform assembly, the key must be inserted in the imprint and then a lateral motion of the second part locks the assembly. This benchmark is inspired from Dechev et al [17].

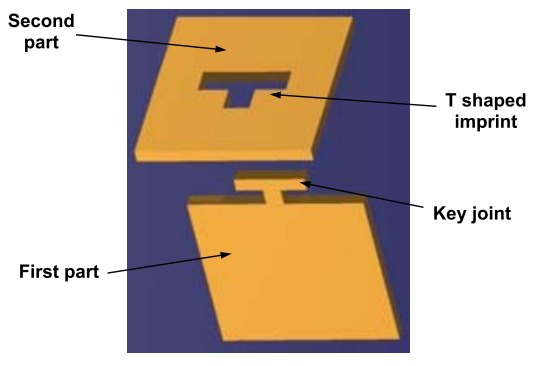

Fig. 8. Lock joint design.

This benchmark has been tested with our robotic structure (figure 9). Parts' orientation is very important, especially for the relative orientation between both micro-objects. The first part is set vertically on the substrate. The gripper is used to grip and align the second part above the key (step 1). When the key is in the imprint (visible on the vertical view), a vertical motion puts the key in the hole (step 2). Finally a lateral motion locks the key and the assembly is performed (step 3).

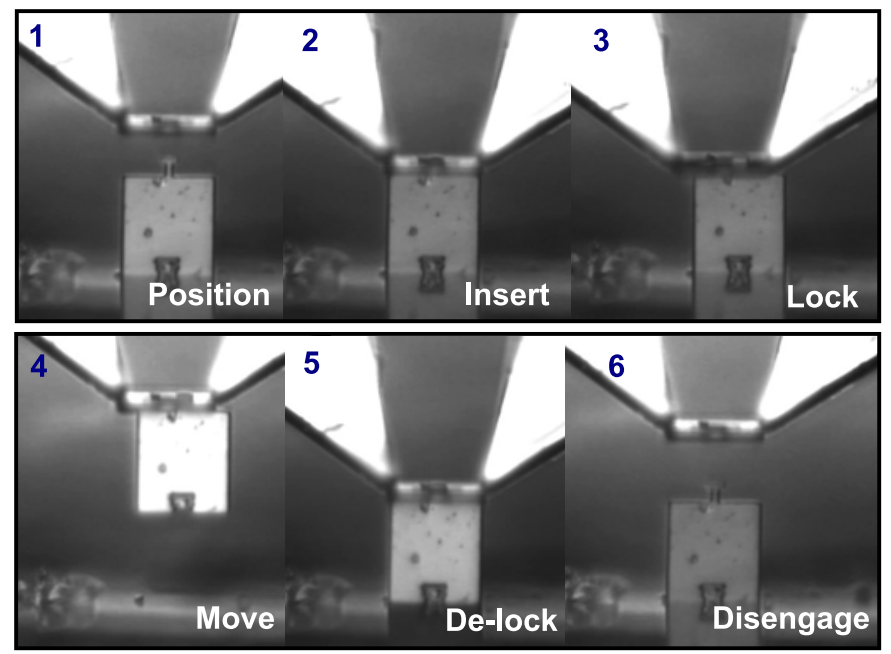

Fig. 9. Reversible assembly.

After locking motion, the 3D microproduct realized can be extracted from the substrate and moved to another place (step 4). Moreover the major interest of this kind of assembly is the possibility to disassemble it. To perform it, motions are repeated on opposite way: a lateral motion to unlock the key (step 5) and a vertical motion to disengage the key from the imprint (step 6). Several cycles of assembly-disassembly have been tested.

\section{Analysis of the reliability}

In order to show the reliability of our method, numerous pick and place operations have been performed in teleoperation and in an automatic cycle. The tests have been done on a silicon micro-objects whose dimensions are $5 \times 10 \times 20 \mu \mathrm{m}^{3}$. The objective of the pick and place operation is to grasp the object placed on the substrate, to move it along $100 \mu \mathrm{m}$ and 
to release it on the substrate. To evaluate the reliability, the success rate of the pick and place operations and the time cycle have been measured.

First, tests have been done in teleoperation. The operator see the lateral view and the vertical view on two screens. He controls the trajectories and the gripper movements with a joystick without force feedback. 60 operations have been done. The time cycle stays always between 3 and 4 seconds. Secondly, tests have been done in an automatic cycle without force and position feedback. The pick and place trajectory was repeated 60 times and the time cycle was 1.8 seconds.

In both tests, the reliability reaches $100 \%$. As only some articles in the litterature quote the reliability of micromanipulation methods, it is quite difficult to compare this value with other works. However, tests of the reliabilty of microhandling strategies have been presented in [19], [20]. Both tests have been done on polystyrene spheres whose diameter is $50 \mu \mathrm{m}$. The success rate was between $51 \%$ and $67 \%$ on around 100 tests in [19] and was between $74 \%$ and $95 \%$ on 60 tests in [20]. Consequently, our method allows a higher reliability on smaller objects which represents a significant contribution.

\section{Discussion}

Based on the several experiments performed with our robotic device, some key points have been highlighted:

Firstly, an adhesive substrate allows reliable release of micro-objects but gripping strength has to be large enough to unstick the handled object. A smart substrate where adhesion could controlled during assembly could be interesting to reduce the gripping force. Moreover, one of the limitation of our method is the difficulties to assemble more than 3 parts in the current configuration. Secondly, micro-objects orientation is very important to perform an assembly. Increasing DOF, especially for rotation motions will improve robotic agility of the device. Thirdly, the microscopic side view is highly useful for teleoperated assembly. Automated assembly could also use this view for visual servoing in addition to the classical close loop control on the vertical view. Fourthly, gripping forces are not directly evaluated. Force sensors will be integrated soon on our silicon end-effectors to perform force controlled gripping tasks.

In a general way, these original experimental tests on generic microparts have opened a lot of new issues and their study will probably brings us to perform reliable microassembly of complex out-of-plane microproducts.

\section{CONCLUSION}

The robotic assembly is one way to produce new microsystems with improved functionalities. We have focused on the study of the assembly of two microparts in order to produce microscopic assembly systems. An original hybrid method between adhesion manipulation and standard gripping has been proposed. Moreover, this method allows to maintain the first object in position with sufficient compliance for assembly. A complete teleoperated robotic structure included micropositioning stages, vision capabilities, piezogripper with silicon end-effectors, has been presented. Two benchmarks of two microparts' assembly have been tested: insertion of object, and locking of object. These experiments have validated our proposed methods and prove the high reliability of this new approach compared to the other methods. Future works will focused on the automation of the assembly.

\section{REFERENCES}

[1] N. Dechev, W.L. Cleghorn, and J.K. Mills. Microassembly of 3-d microstructures using a compliant, passive microgripper. Journal of Microelectromechanical Systems, 13(2):176 189, April 2004.

[2] D. O. Popa and H. E. Stephanou. Micro and meso scale robotic assembly. SME Journal of Manufacturing Processes, 6(1):52-71, 2004.

[3] M. Gauthier, S. Régnier, P. Rougeot, and N. Chaillet. Forces analysis for micromanipulations in dry and liquid media. Journal of Micromechatronics, 3(3-4):389-413, Sept. 2006.

[4] Q. Zhou, B. Chang, and H. N. Koivo. Ambient environment effects in micro/nano handling. In Proc. of the Int. Workshop on Microfactories, pages 146-51, Shangai, China, October 2004.

[5] F. Caruso. Colloids and Colloid Assemblies - Synthesis, Modification, Organization and Utilization of Colloid particles. Wilet-VCH, 2007.

[6] D. H. Gracias, J. Tien, and G. M. Whitesides. Self-assembled electrical networks. United States Patent, US 7007 370, March 7, 2006.

[7] George M. Whitesides and Mila Boncheva. Beyond molecules: Selfassembly of mesoscopic and macroscopic components. Proceedings of the National Academy of Sciences of the United States of America, 99(8):4769-4774, 2002.

[8] Q. Zhou and B. Chang. Microhandling using robotic manipulation and capillary self-alignment. In Intelligent Robots and Systems, 2006 IEEE/RSJ International Conference on, pages 5883-5888, Beijing, China, October 2006.

[9] Fumihito Arai, Hisataka Maruyama, and Toshio Fukuda. 3d manipulation of lipid nanotubes using laser trapped functional gel microbeads. In Proc. of the RSJ/IEEE IROS Conference, pages 3125-3130, San Diego, CA, USA, october 2007.

[10] A. Rosenthal and J. Voldman. Dielectrophoretic traps for singleparticle patterning. Biophysical Journal, 88, march 2005.

[11] M. Gauthier, E. Gibeau, and D. Hriban. Submerged robotic micromanipulation and dielectrophoretic micro-object release. In proc. of the IEEE ICARCV 2006 conference, Singapour, dec. 2006.

[12] F. Dionnet, D. S. Haliyo, and S. Regnier. Autonomous micromanipulation using a new strategy of accurate release by rolling. In Proceedings of the 2004 IEEE ICRA, pages 5019-24, New Orleans, USA, April 2004.

[13] D.S. Haliyo and S. Regnier. Manipulation of micro-objects using adhesion forces and dynamical effects. In Proceedings of ICRA/IEEE Internation Conferenace on Robotics and Automation, May 2002.

[14] C. Clévy, A. Hubert, and N. Chaillet. Flexible micro-assembly system equiped with an automated tool changer. In Journal of Micro-Nano Mechatronics, in press, 2008.

[15] J. Agnus, P. Nectoux, and N. Chaillet. Overview of microgrippers and design of a micromanipulation station based on a mmoc microgripper. In Proc. of IEEE CIRA, Finland, 2005.

[16] David Heriban, Joël Agnus, Jean-René Coudevylle, Michaël Gauthier, and Nicolas Chaillet. Design of silicon finger tips for a moc (microrobot on chip) microgripper. In Proc. of the Int. Workshop on Topica Meeting on Microfactories (TMMF05), Tsukuba, Japan, October 2005.

[17] N. Dechev, J. K. Mills, and W. L. Cleghorn. Mechanical fastener designs for use in the microassembly of $3 \mathrm{~d}$ microstructures. In Proceedings of ASME IMECE 2004, 2004.

[18] F. Arai, D. Andou, Y. Nonoda, T. Fukuda, H. Iwata, and K. Itoigawa. Integrated microendeffector for micromanipulation. In IEEE/ASME Transactions on Mechatronics, volume 3, pages 17-23, 1998.

[19] M. Gauthier, B. Lopez-Walle, and C. Clévy. Comparison between micro-objects manipulations in dry and liquid mediums. In proc. of CIRA'05, June 2005.

[20] Mélanie Dafflon, Benoit Lorent, and Reymond Clavel. A micromanipulation setup for comparative tests of microgrippers. In International Symposium on Robotics (ISR), 2006. 